\title{
Impure Altruism in Dictators' Giving
}

\author{
Oleg Korenok, Edward L. Millner, and Laura Razzolini* \\ Department of Economics \\ Virginia Commonwealth University
}

\begin{abstract}
We design an experiment to test whether incomplete crowding out in dictator games can be rationalized by the impurely altruistic preferences. By giving the recipients an endowment of varying levels, we create an environment in which crowding out may occur. We find that the behavior of 66 percent of the dictators can be rationalized by the impurely altruistic utility function.
\end{abstract}

Keywords: Dictator Game, Impure Altruism, Incomplete Crowding Out

JEL Classifications: C91, D01, D64, H30, H41

\footnotetext{
* We would like to thank Asen Ivanov for patience and many helpful comments and insights. We would also like to thank Rachel Croson, Doug Davis, Catherine Eckel, and participants at the University of Texas, Dallas, the University of Virginia, the University of Kansas, University of Alabama and the 2009 Southern Economics Association seminars, the co-editor, Lise Vesterlund, and three referees for providing helpful and insightful comments on the paper. Research support provided by NSF grant SES-9973731 to Razzolini and the VCU Presidential Research Incentive Fund to Millner is gratefully acknowledged. Any remaining errors are our own. Corresponding author: Oleg Korenok,okorenok@vcu.edu, Richmond VA 23284-4000, (804) 828-3185.
} 


\section{Introduction}

We test whether incomplete crowding out can be rationalized in the dictator game. In the dictator game a subject - the dictator - has the opportunity to share her endowment with another subject - the recipient. Bolton and Katok (1998) report evidence of incomplete crowding out. In their experiment, a \$3 transfer from the dictator to the recipient does not crowd out giving dollar-for-dollar. In particular, 73.7 percent of dictators’ giving is crowded out by the $\$ 3$ transfer.

Evidence from empirical studies and other experiments also demonstrates that crowding out is incomplete. ${ }^{1}$ Many empirical studies (an incomplete list includes Kingma (1989), Okten and Weisbrod (2000), Khanna, Posnett and Sandler (1995), Ribar and Wilhelm (2002), Manzoor and Straub (2005), Hungerman (2005), Borgonovi (2006), Gruber and Hungerman (2007), and Andreoni and Payne (2011)) show that crowding out is quite small, ranging from 0 percent to 30 percent. Payne (1998) reports a somewhat larger but still incomplete crowding out of about 50 percent after controlling for an endogeneity bias. In a public good game, Andreoni $(1993,1995)$ reports that, after a \$2 transfer, the average contribution to a public fund decreased by $\$ 1.43$, or 71percent of \$2, even after controlling for possible subjects’ confusion. Andreoni (1993) concludes that “..., the behavior in the experiment is broadly consistent with the hypothesis that people get pleasure from the act of contributing to the public good” (p. 1325).

The impure altruism model is consistent with incomplete crowding out. This model includes three arguments in the utility function, the dictator's final payoff, the recipient's final payoff and the amount passed by the dictator (Andreoni 1989, 1990). The donor has a taste for giving and derives utility from the very act of passing in addition to the final payoffs. ${ }^{2}$ Consider the situation in which the dictator's endowment is $\$ 10$, the recipient's endowment is $\$ 0$ and the dictator chooses to pass $\$ 5$. That is,

\footnotetext{
${ }^{1}$ One experimental study by Eckel, Grossman and Johnston (2005) reports evidence of complete crowding out when the transfer from the dictator to the recipient is framed as a tax. On the other, when the transfer is neutral, observed crowding out is close to zero.

2 Palfrey and Prisbey $(1996,1997)$ report evidence of a taste for giving in linear public goods games. Also, in the context of the dictator game, Crumpler and Grossman (2008) report that participants donate 20 percent of their endowment even if the amount of money that the charity receives is preset and any contribution by the participant crowds out dollar-for-dollar the giving by the proctor.
} 
passing \$5 maximizes the dictator’s utility. A \$1 transfer from the dictator's endowment to the recipient's endowment, which reduces the dictator's endowment to $\$ 9$ and increases the recipient's endowment to $\$ 1$, should not alter the dictator's optimal choice of equal payoffs if she cares only about the final payoffs, and the dictator should reduce the optimal amount passed to $\$ 4$. However, if the dictator derives utility from passing, the optimal pass would exceed $\$ 4$. After a $\$ 1$ transfer, the dictator decreases her pass by less than $\$ 1$ because she enjoys passing.

We test whether dictators' choices can be rationalized by the impure altruism utility function. To our knowledge, no existence result has been established for the impure altruism model in an equivalent fashion to the Andreoni and Miller (2002) result for the pure altruism model, in which the two final payoffs are the only arguments in the utility function. ${ }^{3}$ To establish this result, Andreoni and Miller examined subjects' choices made on different budget sets and showed that the choices satisfied the Generalized Axiom of Reveal Preferences (GARP). This result, by Afriat's theorem (1967), is equivalent to establishing the existence of a well-behaved utility function.

We modify Andreoni and Miller’s design by giving the recipients an endowment of varying levels to create an environment in which crowding out may occur. We examine subjects' choices made on different budget sets and find that these choices can be rationalized by the impurely altruistic utility function for 66 percent of the dictators. Our study extends Andreoni and Miller's (2002) work by showing that altruistic behavior exhibits a significant degree of rationality in a more general setting that allows for transfers to the recipient and, therefore, the possibility of crowding out.

\section{Experimental Design}

To test whether dictators' choices can be rationalized by a well-behaved impure altruistic utility function, we vary prices as in Andreoni and Miller. We extend their design by also varying the endowments given to both the dictator and the recipient, as in Korenok et al. (2012). This extension creates an environment in which crowding out may occur. The

\footnotetext{
${ }^{3}$ The final payoffs of the dictator and the recipient are the only arguments in a number of other-regarding utility models including Fehr and Schmidt (1999), Bolton and Ockenfels (2000), Charness and Rabin (2002), and Cox and Sadiraj (2010), among others.
} 
result is a dictator game in which the dictator chooses the amount to give, $g$, and the final payoffs to the two subjects are

$$
\begin{aligned}
& \pi_{D}=\left(E_{D}-g\right) p_{h} \\
& \pi_{R}=E_{R}+p_{g} g .
\end{aligned}
$$

In (1), $\pi_{D}$ is the dictator's final payoff, $E_{D}$ is the dictator's endowment, $\pi_{R}$ is the recipient's final payoff, $E_{R}$ is the recipient's endowment, the hold price, $p_{h}$, multiplies the amount that the dictator holds for herself and the price of giving, $p_{g}$, multiplies the amount that the dictator sends to the recipient. ${ }^{4}$ Multiplying the amount passed to the recipient by a price introduces the possibility to tax $\left(\frac{p_{h}}{p_{g}}>1\right)$ or subsidize $\left(\frac{p_{h}}{p_{g}}<1\right)$ the altruistic donation of the dictator. The traditional dictator game occurs when $E_{R}=0$ and $p_{h}$ $=p_{g}=1$.

In the experiment, the subjects are given a menu of choices characterized by different endowments of tokens and different prices, as specified in the first five columns of Table $1 .^{5}$ Each budget in Table 1 specifies the number of tokens to be divided by the dictator, the number of points each token is worth to each subject and the number of points already allocated to the recipient. Consider for example the budget in the second row where $E_{D}=17, E_{R}=11, p_{h}=3$ and $p_{g}=1$. In this case, both the dictator and the recipient have a positive endowment, 17 tokens for the dictator and 11 points for the recipient. Also, given the prices, passing one token reduces by three points the dictator's final payoff and increases by 1 point the recipient's payoff, so that the relative price of giving is 3 .

Table 1: Budgets and Average Dictators’ Choices

\begin{tabular}{cccccccc}
\hline \hline & $\begin{array}{c}\text { Dictator's } \\
\text { Token }\end{array}$ & $\begin{array}{c}\text { Recipient's } \\
\text { Points }\end{array}$ & Hold & Giving & \multicolumn{2}{c}{$\begin{array}{c}\text { Relative } \\
\text { Price of }\end{array}$} & \multicolumn{2}{c}{ Average Tokens Passed } \\
\cline { 5 - 8 } Budget & $\begin{array}{c}\text { Endowment Endowment } \\
\text { Endons 1- }\end{array}$ & Value & Value & Giving & 4 & Sessions 5-6 \\
\hline 1 & 40 & 0 & 1 & 3 & 0.33 & 7.37 & 11.47
\end{tabular}

\footnotetext{
${ }^{4}$ We choose not to attach a price to the recipient's endowment to simplify the dictator's decision with no loss of generality.

5 Building upon Andreoni and Miller's (2002) design, the 18 budgets were constructed so that the prices of passing and holding are integers, and the budget sets would intersect and the intersection realizes substantially below the point where the final payoffs are equal.
} 


\begin{tabular}{cccccccc}
2 & 17 & 11 & 3 & 1 & 3.00 & 3.32 & 2.44 \\
3 & 21 & 2 & 2 & 2 & 1.00 & 4.39 & 5.48 \\
4 & 60 & 0 & 1 & 3 & 0.33 & 10.93 & 16.65 \\
5 & 24 & 12 & 3 & 1 & 3.00 & 4.42 & 4.16 \\
6 & 31 & 2 & 2 & 2 & 1.00 & 6.46 & 7.69 \\
7 & 40 & 0 & 1 & 4 & 0.25 & 6.25 & 10.60 \\
8 & 13 & 12 & 4 & 1 & 4.00 & 2.60 & 1.76 \\
9 & 21 & 2 & 2 & 3 & 0.67 & 3.56 & 5.52 \\
10 & 60 & 0 & 1 & 4 & 0.25 & 10.72 & 17.50 \\
11 & 18 & 12 & 4 & 1 & 4.00 & 3.26 & 2.76 \\
12 & 21 & 3 & 3 & 2 & 1.50 & 4.61 & 4.24 \\
13 & 80 & 0 & 1 & 4 & 0.25 & 13.14 & 23.23 \\
14 & 23 & 12 & 4 & 1 & 4.00 & 4.54 & 2.95 \\
15 & 43 & 6 & 2 & 3 & 0.67 & 6.79 & 10.55 \\
16 & 80 & 0 & 1 & 3 & 0.33 & 14.77 & 18.11 \\
17 & 32 & 16 & 3 & 1 & 3.00 & 5.47 & 3.52 \\
18 & 42 & 4 & 2 & 2 & 1.00 & 8.46 & 10.29 \\
\hline
\end{tabular}

The aim of this study is to determine if the choices made by the subjects when presented with the above 18 budget sets can be rationalized by a well-behaved utility function, $U\left(\pi_{D}, \pi_{R}, g\right)$. In the standard environment, Afriat's (1967) theorem can be applied to the observed data to establish the existence of well behaved preferences that generated those choices. Our budget sets, however, differ substantially from budget sets in the traditional utility theory framework. In the standard framework, given prices and income, a budget set with three goods defines a three-dimensional polyhedron, and the subject's choice of any two goods on the frontier completely identifies the choice of the third. In the context of dictator games, however, there is only one degree of freedom in the subjects' choices. Once $g$ is chosen, then, given endowments and prices, the final payoffs, $\pi_{D}$ and $\pi_{R}$, are completely determined by the system in (1), and satisfy the constraint: $\pi_{D}+\pi_{R} \frac{p_{h}}{p_{g}}=p_{h} E_{D}+\frac{p_{h}}{p_{g}} E_{R} \equiv m$. That is, the budget set corresponds to a line in a three-dimensional space, and the dictator's choice of $g$ completely determines $\pi_{D}$ and $\pi_{R}$ (as shown in Figure 1). The constraints in (1) imply that only one degree of freedom exists in the subjects' choices and that the trade-offs between each pair of the three goods 
$\left(\pi_{D}, \pi_{R}, g\right)$ are not well defined. This means that prices are not well defined, and that the standard application of Afriat's theorem to the observed data is not possible.

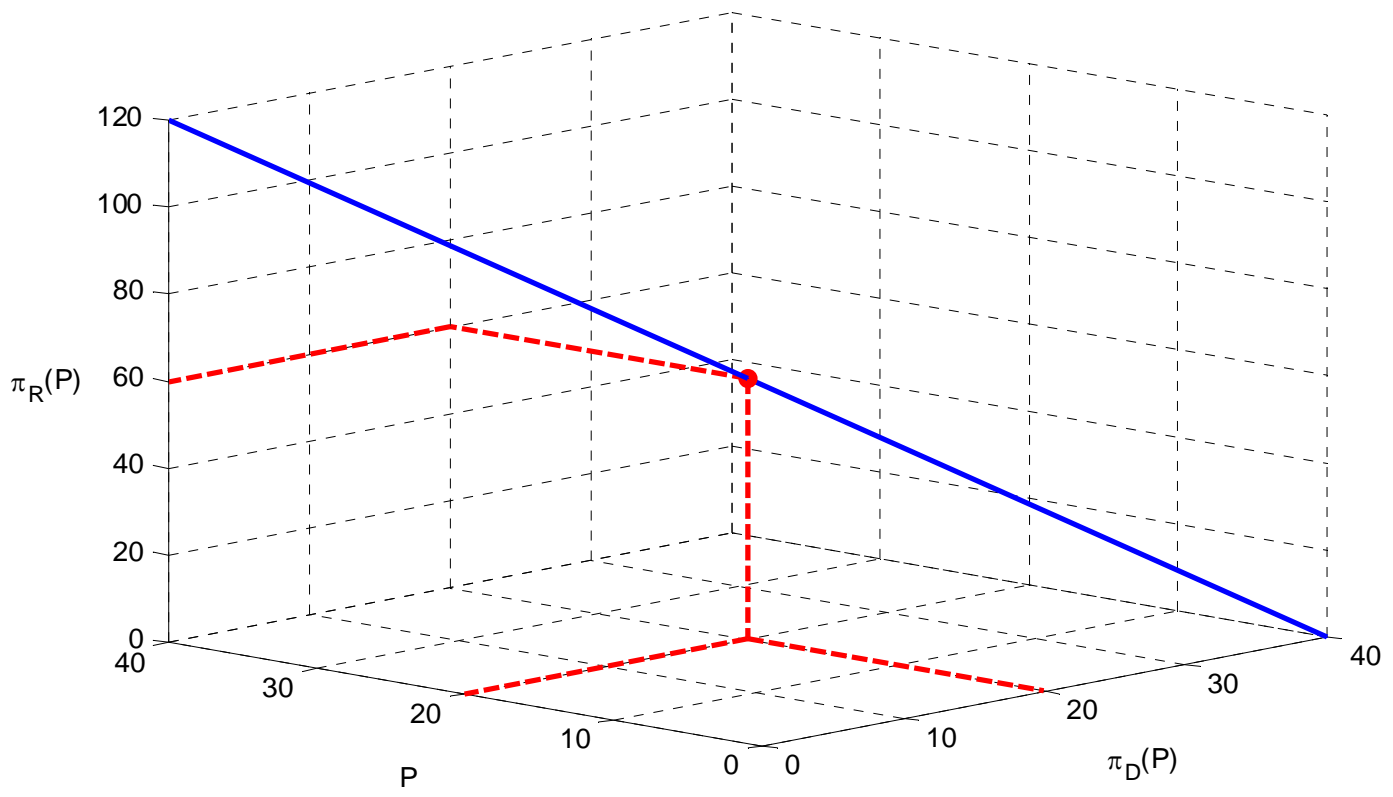

Figure 1: Budget Set in a Modified Dictator Game

\section{Axioms and their Violations}

We develop in this section an alternative argument to establish that observed choices can indeed be rationalized by a well-behaved utility function. Let $x, y, z, \ldots$ be distinct choices or bundles belonging to different budget sets. Using Varian’s (1993) terminology, $x$ is directly revealed preferred to $y$ if $y$ was available in the budget set when $x$ was chosen; $x$ is strictly directly revealed preferred to $y$ if $y$ was available and strictly inside the budget set when $x$ was chosen; and $x$ is revealed preferred to $z$ if $x$ is directly revealed preferred to $y$ and $y$ is directly revealed preferred to $z$.

In the context of our model, when there is one degree of freedom in the choices of the subjects, a trivial modification of the direct revealed preference relation allows us to test whether subjects' choices can be rationalized by a well-behaved utility function. We will say that $x$ is monotonically revealed preferred to $y$ when $x$ is directly revealed preferred to $y^{\prime}$ and $y^{\prime}$ contains more of every good than $y$. Bundle $x$ is indirectly monotonically revealed preferred to $y$ when $x$ is monotonically revealed preferred to $z$ 
and $z$ is monotonically revealed preferred to $y$. Choices $x$ and $y$ are Monotonically Inconsistent if $x$ is monotonically revealed preferred to $y$ and $y$ is monotonically revealed preferred to $x$.

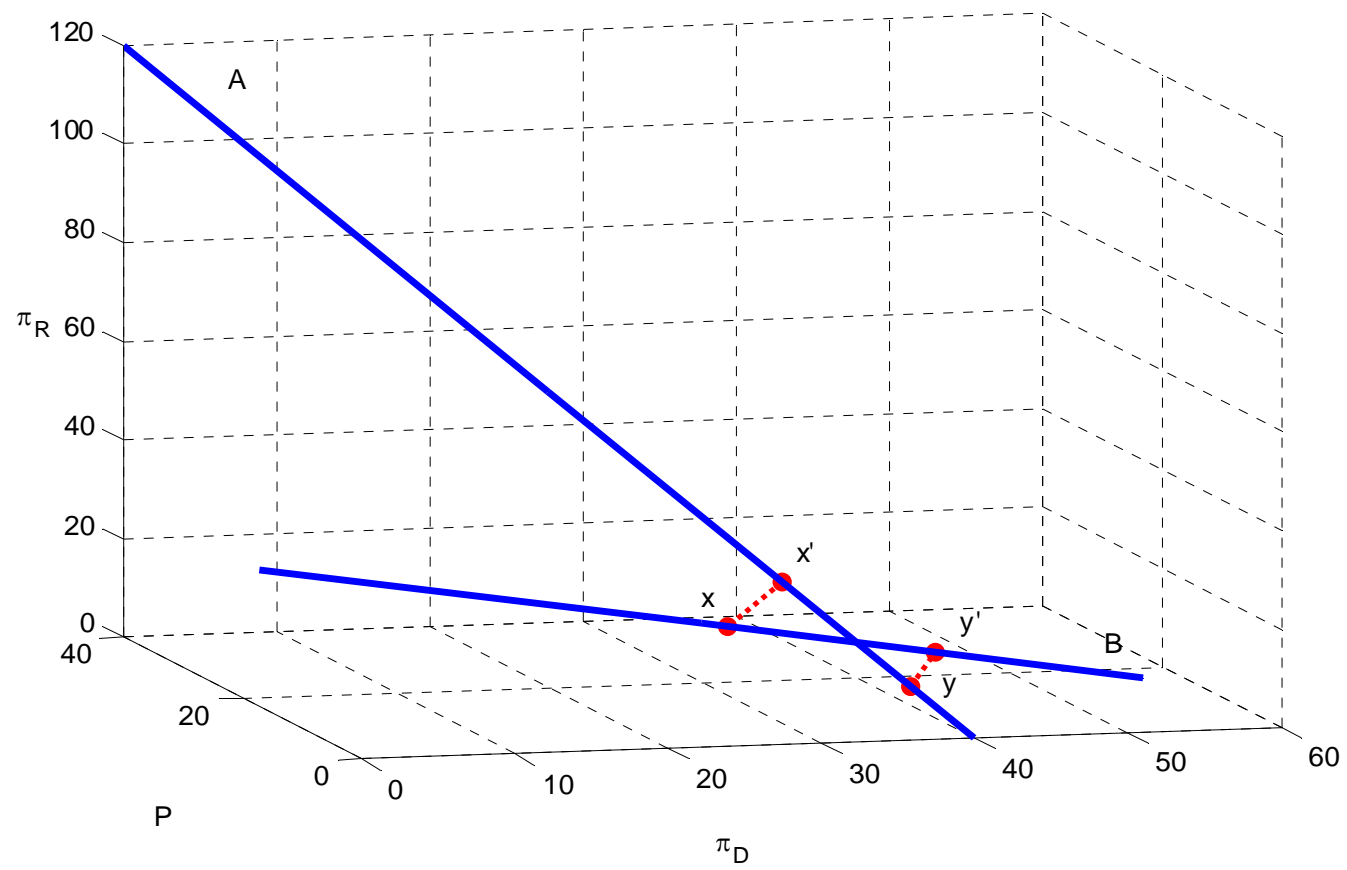

Figure 2: Monotonically Inconsistent Choices in the Space of Payoffs and Pass

Figure 2 provides an example of Monotonically Inconsistent choices in the threedimensional space spanned by the final payoffs and the amount passed. Budget constraint A depicts the first budget in Table 1: $E_{D}=40, E_{R}=0, p_{h}=1, p_{g}=3$. Budget B corresponds to the second: $E_{D}=17, E_{R}=11, p_{h}=3, p_{g}=1$. Let the subject choose bundle $y=(37,9,3)$ when facing budget line A. Since $y$ is chosen, it is directly revealed preferred to bundle $x^{\prime}=(31,27,9)$, which is also available. Bundle $x^{\prime}$ has more of every good than bundle $x=(27,19,8)$. Therefore, $y$ is monotonically revealed preferred to $x$. Similarly, let the subject choose bundle $x$ when facing budget line B. Since $x$ is chosen, it is directly revealed preferred to $y^{\prime}=(39,15,4)$, another point on budget line B. Bundle $y^{\prime}$ has more of every good than $y$. Therefore, $x$ is monotonically revealed preferred to $y$, which establishes that the choices $x$ and $y$ are Monotonically Inconsistent. 
In what follows, we will classify the subjects in our experiment as making Monotonically Inconsistent choices or not. For subjects whose choices are Monotonically Inconsistent we use the following theorem (proof in the Appendix):

Theorem: If the observed choices are Monotonically Inconsistent, no non-satiated utility function exists that could rationalize the choices.

Subjects whose choices are not Monotonically Inconsistent could still be irrational. However, if one can construct a utility function that rationalizes the observed choices, then subjects are rational in the sense that their choices maximize that utility function. We will use the following Result to establish that a well behaved utility function that rationalizes observed choices exists for all subjects in our experiment who are not Monotonically Inconsistent (proof in the Appendix):

Result: If the observed choices are not Monotonically Inconsistent and there exists a set of artificial prices $\bar{p}^{i}$ so that the data are consistent with the Generalized Axiom of Revealed Preferences, then a non-satiated, continuous, concave, monotonic utility function that rationalizes the choices exists.

We check whether subjects' choices are Monotonically Inconsistent under the budget scenarios listed in Table 1. If they are, then using our Theorem, we conclude that a well-behaved utility function, $U\left(\pi_{D}, \pi_{R}, g\right)$, that rationalizes these choices does not exist. If the observed choices are not Monotonically Inconsistent, then we find artificial prices such that the observed choices are consistent with the Generalized Axiom of Revealed Preferences and, using our Result, conclude that a well-behaved utility function that rationalizes the subjects' choices exists.

\section{The Experimental Procedure}

The experiment consisted of 6 sessions conducted in the Experimental Laboratory for Economics and Business Research at Virginia Commonwealth University in the Spring 2009. A total of 178 subjects recruited from introductory economics and business classes participated in the experiment. In sessions 1-4, subjects were randomly assigned by the computer the role of dictator (Blue player) or recipient (Green player). In these sessions, we had 57 dictators and 57 recipients. Subjects earned an average of $\$ 8.98$. We added sessions 5 and 6, in which, as in the Andreoni and Miller's design, each subject was at the 
same time a dictator and a recipient. In these two sessions, we had 62 subjects who earned an average of $\$ 13.06$.

Upon arrival, subjects were randomly seated at computer terminals and given a set of instructions ${ }^{6}$, which were later read aloud by the experimenter. The instructions concluded with a quiz designed to help the participants become familiar with the type of choices involved in the dictator game. The monitor checked the answers to the quiz to make sure that all subjects clearly understood the nature of the choices. After the quiz, in sessions 1-4, the computer randomly determined the role of each subject, either dictator (Blue player) or recipient (Green player).

Throughout the session, no communication between subjects was permitted and all information and choices were transmitted through computer terminals, using the software program z-Tree (Fischbacher, 2007). Each dictator player made simultaneously all 18 decisions specified in Table 1. Next, the dictator's decisions were transmitted by the computer anonymously to the recipient randomly paired with that dictator. After the recipients recorded the 18 decisions on their own personal record sheets, the computer randomly determined which of the 18 decisions to implement and pay out. The subjects recorded their payoffs in a personal log sheet and proceeded to be paid privately by an assistant not involved with the experiment. At this time, the subjects also received a $\$ 5$ participation fee.

\section{Dictators' Rationality}

Our primary goal is to determine whether or not the decisions of the dictators can be rationalized. Before doing so, we examine whether the data in our experiment is consistent with results reported by previous studies. We also examine whether the results in sessions 1-4 differ significantly from the results in sessions 5-6. The analysis provides some external validity to our experiments and suggests that sessions 1-4 and 5-6 may be pooled.

\footnotetext{
${ }^{6}$ Instructions are available at http://www.people.vcu.edu/ /razzolini/instructions.pdf.
} 
We begin by examining whether the data in our experiment are representative of other studies that use the standard dictator game. We find that the results are strikingly similar. Three budgets, 3, 6, and 18, are relatively close to budgets presented in the standard dictator game. Under these budgets, the relative price of giving equals 1 and the recipient's endowment is quite small, less than 10 percent of the dictator's endowment. The last two columns of Table 1 report the average number of tokens passed in sessions 1-4 and 5-6 for each budget set. The percentages of tokens passed in session 1-4 under budgets 3, 6, and 8 are 21 percent, 21 percent, and 22 percent, respectively. The percentages of tokens passed in session 5-6 under budgets 3, 6, and 8 are 26 percent, 25 percent, and 25 percent, respectively. The average across all three budgets and six sessions is 23 percent, which is quite close to the pass rates reported in the literature for the standard game. ${ }^{7}$

Next we compare our results with those reported by Andreoni and Miller for two identical budgets. We again find similarity in the results. Budgets 1 and 7 in our experiment are identical to budgets 2 and 11 in Andreoni and Miller. The average amounts passed under budgets 1 and 7 in sessions 5-6 of our experiments are 11.47 and 10.60 , respectively. The average amounts passed under budgets 2 and 11 of their experiments are 12.80 and 14.80 , respectively.

Can sessions 1-4 be pooled with sessions 5-6? We are unable to find significant differences in the choices using two different tests, and conclude that pooling the six sessions is appropriate. We use the Mann-Whitney test to compare the median amounts passed under each budget. We prefer to compare medians rather than means because heterogeneity of dictators' giving results in highly non-symmetric and multimodal distributions of the amount passed. The median amounts are identical for 8 budgets and they are never significantly different at the 5 percent level. We also compare the distribution of the amounts passed using the Kolmogorov-Smirnov test. Again, for all 18 budgets, the distributions in sessions 1-4 do not significantly differ from the distributions in sessions 5-6 at the 5 percent significance level.

\footnotetext{
${ }^{7}$ See, for a discussion, the excellent survey by Camerer (2003).
} 
We are now prepared to address directly the primary question of the paper: can dictators' giving be rationalized by a well-behaved impure altruism utility function of the form $U\left(\pi_{D}, \pi_{R}, g\right)$ ? We find that 78 (66 percent) of our 119 dictators never make Monotonically Inconsistent choices. We then identify artificial prices that make those choices consistent with the Generalized Axiom of Revealed Preference and, using our Result, conclude that a well-behaved utility function that rationalizes the subjects’ choices exists. ${ }^{8}$ Table 2 contains information about the 41 subjects whose choices are Monotonically Inconsistent. The first three columns of Table 2 list the sessions, the subjects with inconsistent choices and the number of inconsistent choices. ${ }^{9}$

Because we gave an endowment to the recipient, the dictator's choices of the final allocation are constrained. Even a selfish dictator cannot reduce the recipient's final payoff to zero. As a result, violations could be created or masked by this constraint. To account for this, when checking for MIC violations, we moved all constrained choices along the budget line to the point where the recipient’s final payoff equals zero. To illustrate in two dimensions, Figures 3 (a) and (b) show how an observed constrained choice at point $x$ is moved to the unconstrained choice $x$ '.

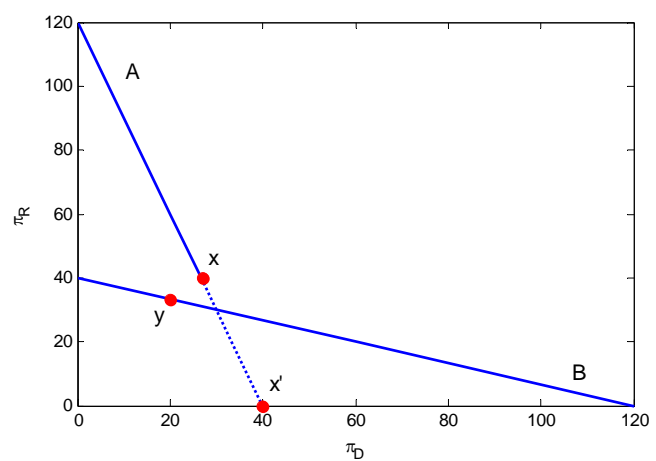

3(a)

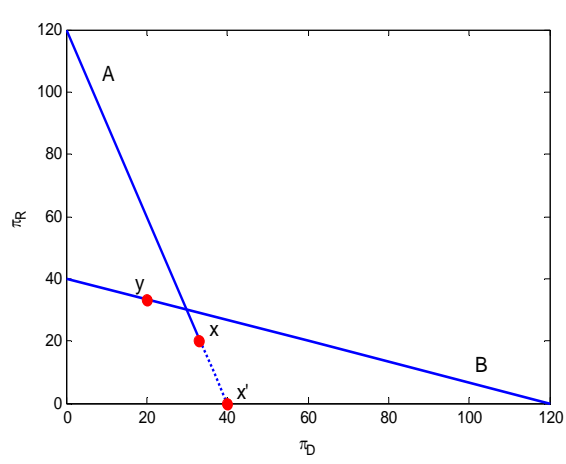

3(b)

Figure 3: Violations with Constrained and Unconstrained choices ${ }^{10}$

\footnotetext{
8 The set of artificial prices is available upon request. Note that prices were found for each subject that did not make any monotonically inconsistent choice. Since the prices are not real, the choices satisfy GARP in an artificial sense.

${ }^{9}$ For any two budget sets, the maximum number of possible violations is one. For any three budget sets, the maximum number of violations is four, equal to the number of possible budget pairs plus the budgets' triple. Continuing in this fashion, for any 18 budget sets, the maximum number of possible violations is equal to $2^{18}-18=262,126$, the sum of all possible pairs, triples and so on.

${ }^{10}$ We thank an anonymous reviewer for suggesting the use of this graphic illustration.
} 
Figure 3(a) shows how a truncated budget set may mask a violation. Suppose a selfish subject when facing budget line A in Figure 3(a) chooses point $x$, while he chooses point $y$ when facing budget B. These observed choices do not constitute a MIC violation. Moving choice $x$ to $x$ ' creates an additional violation, not previously observed. On the other hand, Figure 3(b) shows that a truncated budget does not necessarily mask a violation. Choices $x$ and $y$ already constitute a violation and moving the constrained choice $x$ to the unconstrained choice $x$ ' does not create an additional violation. Truncated budget sets, also, may or may not create additional violations.

To check if truncated budget sets mask violations or create additional ones, we looked for MIC violations after moving all constrained choices along the budget line to the point where the recipient's payoff equals zero. This procedure did not affect the number or severity of observed violations; nor did it affect the number and identity of dictators whose choices are rationalizable. Thus, truncated budget sets do not drive our results.

Table 2: Monotonically Inconsistent Choices (MIC)

\begin{tabular}{cccc}
\hline \hline & Subject & Number of MIC & $\begin{array}{c}\text { Critical Cost } \\
\text { Effic. Index* }\end{array}$ \\
\hline Sessions 1-4: & 1 & 1 & 0.99 \\
& 2 & 2 & $1.00^{*}$ \\
& 8 & 2 & $1.00^{*}$ \\
& 10 & 15 & 0.97 \\
15 & 22 & 0.87 \\
16 & 1 & $1.00^{*}$ \\
17 & 7 & $1.00^{*}$ \\
18 & 19 & 0.80 \\
19 & 7 & 0.97 \\
34 & 14 & 0.72 \\
& 36 & 1 & $1.00^{*}$ \\
37 & 14 & 0.83 \\
& 12 & 1 & $1.00^{*}$ \\
& 50 & 11 & 0.95 \\
51 & 6 & $1.00^{*}$ \\
54 & 4 & 0.93 \\
57 & & 0.95
\end{tabular}




\begin{tabular}{|c|c|c|c|}
\hline Sessions 5-6: & 60 & 24 & 0.90 \\
\hline & 62 & 1 & $1.00^{*}$ \\
\hline & 64 & 6 & 0.93 \\
\hline & 66 & 7 & 0.97 \\
\hline & 67 & 2 & 0.89 \\
\hline & 75 & 9 & $1.00^{*}$ \\
\hline & 77 & 36 & 0.83 \\
\hline & 78 & 5 & 0.95 \\
\hline & 81 & 2 & 0.96 \\
\hline & 85 & 3 & 0.95 \\
\hline & 87 & 14 & 0.92 \\
\hline & 92 & 3 & 0.95 \\
\hline & 93 & 16 & 0.77 \\
\hline & 94 & 8 & 0.93 \\
\hline & 99 & 3 & $1.00^{*}$ \\
\hline & 101 & 17 & 0.92 \\
\hline & 104 & 6 & 0.83 \\
\hline & 105 & 27 & 0.83 \\
\hline & 106 & 1 & $1.00^{*}$ \\
\hline & 107 & 8 & 0.99 \\
\hline & 109 & 1 & 0.96 \\
\hline & 115 & 2 & 0.95 \\
\hline & 117 & 1 & $1.00^{*}$ \\
\hline & 119 & 13 & 0.90 \\
\hline
\end{tabular}

Note: * Indicates that an $\varepsilon$-change eliminates all MIC choices.

Few dictators make severely Monotonically Inconsistent choices. As in Andreoni and Miller (2002), we use a modification of Afriat's (1972) Critical Cost Efficiency Index (CCEI) to measure the severity of inconsistencies. The index shows the largest value by which the dictator's endowments can be multiplied to eliminate all inconsistencies. ${ }^{11}$ The difference between 1 and the CCEI can be interpreted as the

\footnotetext{
${ }^{11}$ For each subject and budget line, we find whether changing the ith budget line from the one defined by $m=E_{D_{i}} p_{h_{i}}+E_{R_{i}} \frac{p_{h_{i}}}{p_{g_{i}}}$ to the one defined by $m^{\prime}=t E_{D_{i}} p_{h_{i}}+E_{R_{i}} \frac{p_{h_{i}}}{p_{g_{i}}}$ eliminates any occurrences of MIC. We find the CCEI by decreasing t until the sequence of 18 shifts of the budget lines eliminates each occurrence of MIC. For example, consider subject 1 in Table 2. When we multiply his/her endowment in
} 
proportion of the endowment that the dictator 'wastes' on his most inconsistent choice. The last column in Table 2 reports the index. Only 16 (13 percent) of the dictators have a CCEI below 0.95 (the threshold adopted by Varian (1991)). These 16 subjects appear to be 'wasting' more than 5 percent of their endowment.

Our revealed preference tests are quite powerful according to Bronars’ (1987) test (for a detailed discussion of various measures of the power of revealed preferences tests see Andreoni and Harbaugh (2005)). Ex-ante, our experiment offers many opportunities to display an inconsistent behavior. To show this, we generate artificial choices for 50,000 subjects by randomly drawing points from a uniform distribution over each budget set. The vast majority of these simulated subjects, 94.7 percent, make Monotonically Inconsistent choices at least once, with an average of 23.40 inconsistencies, and 80 percent have a CCEI below 0.95. Ex-post, dictators also make choices that could lead to many inconsistencies. To demonstrate this, we generate artificial choices for 50,000 subjects by randomly drawing from the set of actual choices made in the experiment. Even more of these bootstrapped subjects, 97.7 percent, make Monotonically Inconsistent choices at least once, with an average of 24.52 inconsistencies.

A natural question to ask is whether adding the amount passed as a third argument in the utility function is necessary. That is, can the behavior of the dictators be rationalized by the purely altruistic utility function, $U\left(\pi_{D}, \pi_{R}\right)$ ? If we limit our analysis to the final payoff space, $\left(\pi_{D}, \pi_{R}\right)$ and replicate Andreoni and Miller's methodology on our data, extending when necessary the truncated budget sets, 58 (49 percent) of the 119 dictators violate GARP at least once, and 38 (32 percent) have severe violations with a CCEI below 0.95. On the other hand, 10 percent of the subjects in the Andreoni and Miller experiment violate the axiom at least once, and only 2 percent have severe violations. Thus, we find that the third argument is crucial when crowding out is possible.

The fact that in our design the recipients' endowments take positive values in 12 out of 18 decisions explains why our results differ from Andreoni and Miller's results. Incomplete crowding out is possible only when the recipient's endowment varies and 
may lead to violations of the revealed preference axioms in the final payoffs' space. Andreoni and Miller did not detect violations of the axioms in the final payoff space due to incomplete crowding because they never gave recipients a positive endowment. ${ }^{12}$

\section{Estimating Individual Preferences to Predict Out-of-Sample Behavior}

Having established that the impure altruism utility function, $U\left(\pi_{D}, \pi_{R}, g\right)$, rationalizes the choices for most of the dictators, we next determine its form and estimate its parameters to make out-of-sample predictions. We find that the impure altruism model provides fairly accurate predictions for the experiment conducted by Bolton and Katok (1998).

Following Andreoni and Miller (2002) and the replication of their experiment by Fisman, Kariv, and Markovits (2007), we begin by estimating the parameters of a Constant Elasticity of Substitution utility function

$$
U\left(\pi_{D}, \pi_{R}, g\right)=\left(\alpha \pi_{D}^{\rho}+\beta \pi_{R}^{\rho}+(1-\alpha-\beta) g^{\rho}\right)^{\frac{1}{\rho}}
$$

Parameters $\alpha$ and $\beta$ are the relative weights on the payoff to the dictator and the recipient, respectively. The CES function incorporates a number of other utility functions as special cases as the parameter $\rho$ varies. As $\rho$ approaches -1 , utility converges to perfect substitutes preferences: $U\left(\pi_{D}, \pi_{R}, g\right)=\alpha \pi_{D}+\beta \pi_{R}+(1-\alpha-\beta) g$; as $\rho$ approaches $\rightarrow$, utility converges to Leontief preferences: $U\left(\pi_{D}, \pi_{R}, g\right)=\min \left\{\alpha \pi_{D}, \beta \pi_{R},(1-\alpha-\beta) g\right\}$; and as $\rho$ approaches 0 , utility converges to the Cobb-Douglas preferences: $U\left(\pi_{D}, \pi_{R}, g\right)=\pi_{D}^{\alpha} \pi_{R}^{\beta} g^{(1-\alpha-\beta)}$.

Maximizing the CES function subject to the budget constraints in (1) yields the following first order condition:

$$
\alpha\left(\left(E_{D}-g\right) p_{h}\right)^{\rho-1}-\beta\left(E_{R}+p_{g} g\right)^{\rho-1}-(1-\alpha-\beta) g^{\rho-1}=0,
$$

which we solve numerically for the optimal give level, $g\left(E_{D}, E_{R}, p_{h}, p_{p} ; \alpha, \beta, \rho\right)$. As in Fisman, Kariv, and Markovits (2007), we specify the econometric model for individual dictators in terms of budget shares:

\footnotetext{
${ }^{12}$ The results in this section are robust to using the value of the amount passed to the recipient, $p_{g} g$, rather than the number of tokens passed, $g$, as an argument in the utility function, that is, using $U\left(\pi_{D}, \pi_{R}, p_{g} g\right)$ rather than $U\left(\pi_{D}, \pi_{R}, g\right)$. These results are available upon request.
} 


$$
\frac{\pi_{D, i}^{n}}{m^{n}}=\frac{\left(E_{D}^{n}-g_{i}\left(E_{D}^{n}, E_{R}^{n}, p_{h}^{n}, p_{g}^{n} ; \alpha_{i}, \beta_{i}, \rho_{i}\right)\right) p_{h}^{n}}{m^{n}}+\epsilon_{i}^{n}, \quad n=1, \ldots, 18
$$

where $g_{i}\left(E_{D}^{n}, E_{R}^{n}, p_{h}^{n}, p_{g}^{n} ; \alpha_{i}, \beta_{i}, \rho_{i}\right)$ is the optimal give for subject $i$ under budget $n, \epsilon_{i}$ are i.i.d. normally distributed errors with mean zero and variance $\sigma_{i}^{2}$, and $m^{n}=\pi_{D}^{n}+\frac{p_{h}^{n}}{p_{g}^{n}} \pi_{R}^{n}$. Since $0 \leq \frac{\pi_{D, i}^{n}}{m^{n}} \leq 1$, the distribution for $\epsilon_{i}$ is truncated. We estimate $\alpha_{i}, \beta_{i}, \rho_{i}$ and $\sigma_{i}^{2}$ via a non-linear two-limit Tobit maximum likelihood. Prior to estimation we omit 30 subjects with uniformly selfish choices, 1 subject who chose to equalize final payoffs in all 18 decisions (Leontief), and 2 subjects that uniformly chose to maximize the sum of final payoffs (perfect substitutes).

For the remaining 86 subjects (72 percent), we estimate the parameters using the CES specification. For 53 subjects, the sum $\hat{\alpha}_{i}+\hat{\beta}_{i}$ is approximately one; for 52 of these 53 subjects $\hat{\rho}_{i}<-0.5$. A Monte-Carlo study we conducted confirmed that parameters $\alpha_{i}$ and $\beta_{i}$ are poorly identified when the true $\rho<-0.5$. To address this problem we reestimated the parameters for all 52 subjects with $\hat{\rho}_{i}<-0.5$, restricting the CES to Leontief preferences. We also re-estimated the parameters for 32 subjects for whom $\hat{\rho}_{i}$ was within three standard deviations from 0, restricting the CES to Cobb-Douglas preferences. For those subjects for whom we estimated both a Leontief and a CobbDouglas function, we use estimates for the specification with the smallest root-mean squared error.

We test whether adding the amount passed as a third argument in the utility function is necessary. That is, can the purely altruistic utility function, $U\left(\pi_{D}, \pi_{R}\right)$, fit the data as well? The likelihood ratio test rejects the null hypothesis of two arguments in the utility function for 38 (32 percent) of the 119 dictators at 5\% significance level. That is, for one third of the subjects we observe significant incomplete crowding out. 
We can predict the Bolton and Katok (1998) results with an impressive accuracy using the estimates generated from all 119 subjects. ${ }^{13}$ Bolton and Katok (1998) examined dictators' giving under two treatments: treatment 18-2 in which $E_{D}=\$ 18, E_{R}=\$ 2$, $p_{h}=\$ 1, p_{g}=\$ 1$, and treatment 15-5, in which $E_{D}=\$ 15, E_{R}=\$ 5, p_{h}=\$ 1, p_{g}=\$ 1$. Compared to treatment 18-2, in treatment 15-5 the experimenter transfers $\$ 3$ from the dictator to the recipient. Our estimates suggest that many dictators care not only about final payoffs, but also about the amount passed and, thus, crowding out will be incomplete. We predict the amount passed for each of our 119 dictators under both treatments. The average amount passed falls from $\$ 3.65$ in the 18-2 treatment to $\$ 2.41$ in the 15-5 treatment. Our prediction is close to Bolton and Katok's results, as they found that the average amount passed fell from $\$ 3.48$ in the 18-2 treatment to $\$ 2.62$ in the 15-5 treatment.

\section{Conclusion}

Our research extends the work of Andreoni and Miller (2002) and Fisman, Kariv, and Markovits (2007) in establishing that unselfish behavior meets economists' definition of rationality and that a standard, testable utility maximization model fits well the evidence from the laboratory and from the field. Following the approach of Andreoni and Miller's seminal paper, we show that dictator's choices can be rationalized by the impure altruism utility function, $U\left(\pi_{D}, \pi_{R}, g\right)$. The behavior of 66 percent of the dictators in our experiment can be rationalized with the impurely altruistic utility function. Such a utility function can rationalize incomplete crowding out “...the oldest and the most important question in public economics” (Andreoni and Payne, 2009, p.1).

Our results confirm the importance of recognizing that individuals attach value to the act of giving. If the government were to reduce its contributions to charitable organizations and simultaneously decrease taxes by the same amount with a balanced budget fiscal policy, incomplete crowding out predicts a decrease in charitable giving, while complete crowding out predicts no change in charity revenue. Alternatively, if the

\footnotetext{
13 To generate predictions, for 86 subjects we use the impure altruistic parameters estimates, for 30 subjects we use the selfish prediction, for two subjects we use perfect substitute and for one subject Leontieff preferences.
} 
government were to increase its contributions with a balanced budget fiscal policy, charity revenue will increase. More importantly, we establish that such incomplete crowding out behavior is rational.

\section{Appendix}

Let the vector $x$ denote the triple $\left(\pi_{D}, \pi_{R}, g\right)$ of observed choices. Let $x^{i}$ be a chosen triple under given values of $E_{D}^{i}, E_{R}^{i}, p_{H}^{i}, p_{g}^{i}$, for $i=1, \ldots, n$. Let the set of all $x$ such that $x \in R_{3}^{+}$: $x<x^{j}$ or $x=x^{j}$, where $x^{j} \in R_{3}^{+}: \pi_{D}^{i}+\frac{p_{h}^{i}}{p_{g}^{i}} \pi_{R}^{i}=\pi_{D}^{j}+\frac{p_{h}^{i}}{p_{g}^{i}} \pi_{R}^{j}$ define a polyhedron $S^{i}$ as depicted in blue in Figure 3. We will say that the utility function $U\left(\pi_{D}, \pi_{R}, g\right)=U(x)$ rationalizes the set of $n$ observations $\left(x^{i}\right)$ if $U\left(x^{i}\right) \geq U(x)$ for all $x \in S^{i}$.

Theorem: If the observed choices are Monotonically Inconsistent, no non-satiated utility function exists that could rationalize the choices.

Proof: Let $x^{i}$ and $x^{j}$ be two monotonically inconsistent choices. By definition of Monotonically Inconsistency, $x^{i}$ is monotonically revealed preferred to $x^{j}$ and $x^{j}$ is monotonically revealed preferred to $x^{i}$. This implies that $x^{i}$ is directly revealed preferred to $\hat{x}^{j}$ and $\hat{x}^{j}$ contains more of every good than $x^{j}$, or $x^{j} \in S^{i}$. Similarly $x^{j}$ is directly revealed preferred to $\hat{x}^{i}$ and $\hat{x}^{i}$ contains more of every good than $x^{i}$, or $x^{i} \in S^{j}$. Assume to the contrary that a nonsatiated utility function exists and it rationalizes the observed choices $x^{i}$ and $x^{j}$. By definition of rationalization, $x^{j} \in S^{i}$ implies that $U\left(x^{i}\right) \geq U\left(x^{j}\right)$. If $U\left(x^{i}\right)=U\left(x^{j}\right)$, by local nonsatiation there exists an $\bar{x}$ such that $\bar{x} \in S^{i}$ and $U(\bar{x})>U\left(x^{j}\right)=U\left(x^{i}\right)$. But then $U(x)$ cannot rationalize $x^{i}$ because there is $\bar{x} \in S^{i}$ such that $U(\bar{x})>U\left(x^{i}\right)$. Then, it must be $U\left(x^{i}\right)>U\left(x^{j}\right)$. Similarly, we can show that $x^{i} \in S^{j}$ implies that $U\left(x^{j}\right)>U\left(x^{i}\right)$. This contradicts our previous claim that $U\left(x^{i}\right)>U\left(x^{j}\right)$.

To establish the Result, given any triple $x^{i}$ we construct an artificial set of strictly positive prices $\bar{p}^{i}$ such that $\bar{p}^{i} x^{i}=\left(1, \frac{p_{h}^{i}}{p_{g}^{i}}, 0\right) x^{j}$, where $x^{j} \in R_{3}^{+}: \pi_{D}^{i}+\frac{p_{h}^{i}}{p_{g}^{i}} \pi_{R}^{i}=\pi_{D}^{j}+\frac{p_{h}^{i}}{p_{g}^{i}} \pi_{R}^{j}$. 
Let the set $\bar{S}^{i}$ be defined as $\bar{S}^{i}=\left\{\bar{x} \in R_{3}^{+}: \bar{p}^{i} x^{i} \geq \bar{p}^{i} \bar{x}\right\}$, depicted in green in Figure 3 . Notice that by construction $S^{i} \subset \bar{S}^{i}$.

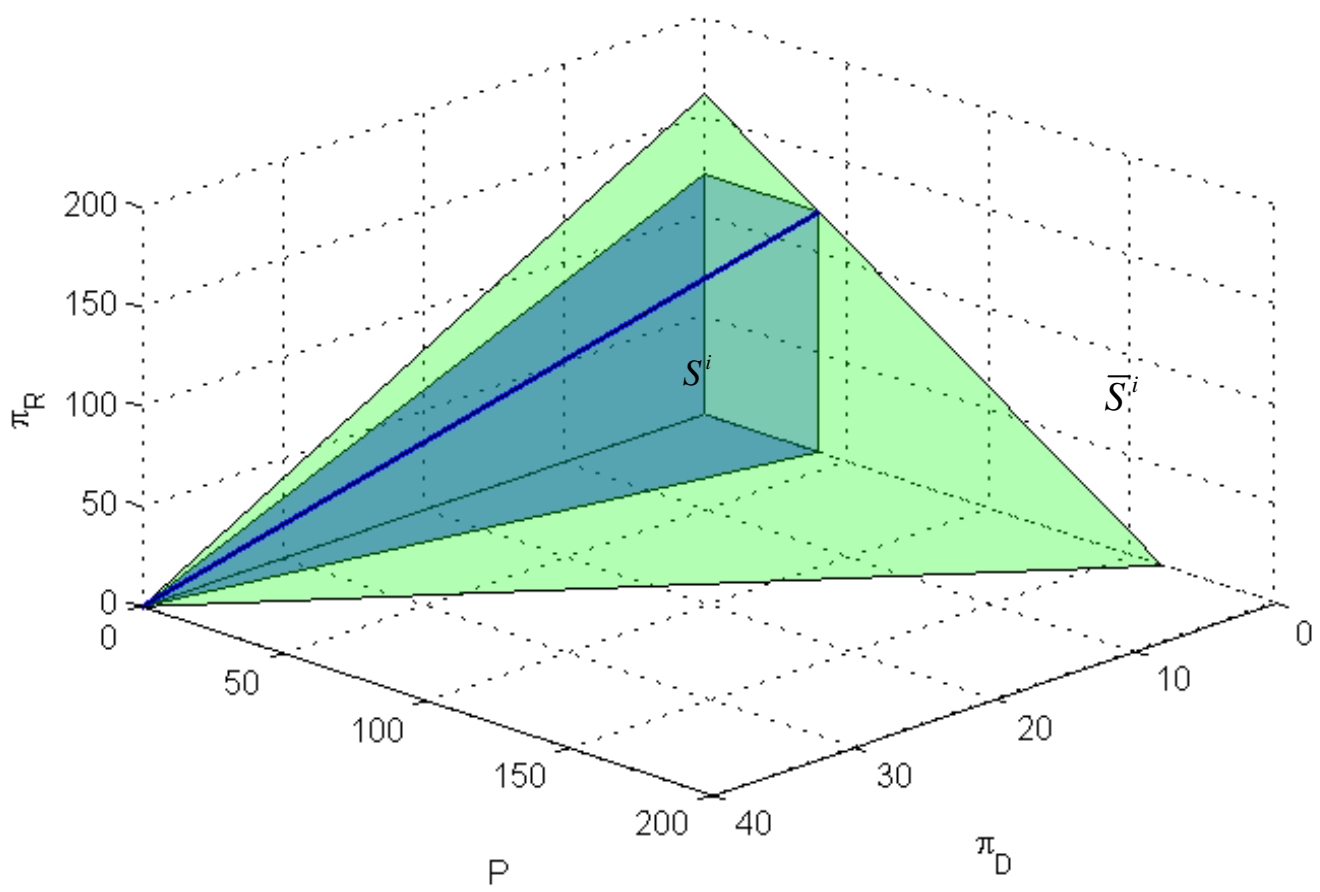

Figure 3: Geometry of $S$ and $\bar{S}$

Result: If the observed choices are not Monotonically Inconsistent and there exists a set of artificial prices $\bar{p}^{i}$ so that the data are consistent with the Generalized Axiom of Revealed Preferences, then a non-satiated, continuous, concave, monotonic utility function that rationalizes the choices exists.

Proof: Let $x^{i} i=1, \ldots, n$ be a set of observations that are not Monotonically Inconsistent, $x^{i} \in S^{i} \subset \bar{S}^{i}$ for all $i$. Afriat's theorem can be applied to the set $\bar{S}^{i}$. If the observations $x^{i}$ satisfy GARP, then by Afriat's theorem there exists a well behaved utility function $U(x)$ that rationalizes the choices, so that $U\left(x^{i}\right) \geq U(x)$ for all $x$ such that $\bar{p}^{i} x^{i} \geq \bar{p}^{i} x$ or $x \in \bar{S}^{i}$. Since $S^{i} \subset \bar{S}^{i}, U\left(x^{i}\right) \geq U(x)$ for all $x \in S^{i}$. This implies that the same utility function rationalizes the individual's choices over the polyhedron $S^{i}$. 


\section{Bibliography}

Afriat, S. (1967). "The construction of a utility function from expenditure data." International Economic Review, 8, 67-77.

Afriat, S. (1967). "Efficiency Estimation of production functions.” International Economic Review, 13, 568-598.

Andreoni, J. (1989). "Giving with Impure Altruism: Applications to Charity and Ricardian Equivalence.” The Journal of Political Economy, 97(6), 1447-58.

Andreoni, J. (1990). "Impure altruism and donations to pubic goods: A theory of warm glow giving.” The Economic Journal, 100, 464-77.

Andreoni, J. (1993). “An Experimental Test of the Public-goods Crowding-out Hypothesis.” American Economic Review, 83, 1317-1327.

Andreoni, J. (1995). "Cooperation in Public-Goods Experiments: Kindness or Confusion?" American Economic Review, 85(4), 891-904.

Andreoni, J. and W. T. Harbaugh (2005). "Power indices for revealed preference tests," Working papers 10, Wisconsin Madison - Social Systems.

Andreoni, J., and J. Miller (2002). "Giving according to GARP: an experimental test of the consistency of preferences for altruism.” Econometrica, 70, 737-53.

Andreoni, J. and A. A. Payne (2011). "Is Crowding Out Due Entirely to Fundraising? Evidence from a Panel of Charities.” Journal of Public Economics, 95(5-6), 33443.

Bolton, G.E., and E. Katok (1998). "An experimental test of the crowding out hypothesis: The nature of beneficent behavior," Journal of Economic Behavior and Organization, 37(3), 315-31.

Bolton, G.E., and A. Ockenfels (2000). "ERC: A theory of equity, reciprocity, and competition.” The American Economic Review, 90(1), 166-93.

Borgonovi, F. (2006). "Do Public Grants to American Theatres Crowd-out Private Donations?” Public Choice, 126, 429-451. 
Bronars, S.G. (1987). "The Power of nonparametric tests of preference maximization," Econometrica, 55, 693-98.

Camerer, C. F., 2003. Behavioral game theory: experiments in strategic interaction. Princeton: Princeton University Press.

Charness, G., and M. Rabin, (2002).“Understanding Social Preferences with Simple Tests.” Quarterly Journal of Economics, 117, 817-869.

Cox, J.C., and V. Sadiraj (2010). "Direct Tests of Models of Social Preferences." Economic Inquiry, forthcoming.

Crumpler, H. and P.J. Grossman (2008). "An Experimental Test of Warm Glow Giving,” Journal of Public Economics, 92, 1011-1021.

Eckel, C. C., P.J. Grossman and R. M. Johnston (2005). "An experimental test of the crowding out hypothesis,” Journal of Public Economics, 89, 1543-1560.

Fehr, E., and K. M. Schmidt (1999). "A theory of fairness, competition and cooperation.” Quarterly Journal of Economics, 114, 817-68.

Fischbacher, U. (2007). "z-Tree: Zurich Toolbox for Ready-Made Economic Experiments,” Experimental Economics. 10, 171-178.

Fisman, R., S. Kariv and D. Markovits (2007). "Individual preferences for giving.” The American Economic Review, 97(5), 1858-1876.

Gruber, J. and D.M. Hungerman (2007). "Faith-based Charity and Crowd-out During the Great Depression” Journal of Public Economics, 91, 1043-1069.

Hungerman, D.M. (2005). “Are Church and State Substitutes? Evidence from the 1996 Welfare Reform.” Journal of Public Economics, 89, 2245-2267.

Khanna, J., J. Posnett, and T. Sandler (1995). "Charity Donations in the UK: New Evidence Based on Panel Data.” Journal of Public Economics, 56, 257-272.

Kingma, B. (1989). "An Accurate Measure of the Crowd-Out Effect, Income Effect, and Price Effect for Charitable Contributions," Journal of Political Economy, 97, 1197-1207.

Korenok, O., E.L. Milner, and L. Razzolini (2012) “Are Dictators Averse to Inequality,” Forthcoming, Journal of Economic Behavior and Organization. 
Manzoor, S.H. and J.D. Straub (2005). “The Robustness of Kingma’s Crowd-out Estimate: Evidence from New Data on Contributions to Public Radio.” Public Choice, 12,463-476.

Okten, C. and B.A. Weisbrod (2000). "Determinants of Donations in Private Nonprofit Markets.” Journal of Public Economics, 75, 255-272.

Palfrey, T.R., and J.E. Prisbrey (1996). "Altruism, Reputation and Noise in Linear Public Goods Experiments.” Journal of Public Economics, 61, 409-427.

Palfrey, T.R., and J.E. Prisbrey (1997). “Anomalous Behavior in Public Goods Experiments: How Much and Why?” The American Economic Review, 87(5), 829-847.

Payne, A. A. (1998). "Does the Government Crowd Out Private Donations?: New Evidence from a Sample of Nonprofit Firms.” Journal of Public Economics, 69, 323-345.

Ribar, D.C. and M.O.Wilhelm (2002). "Altruistic and Joy-of-Giving Motivations in Charitable Behavior.” Journal of Political Economy, 110, 425-457.

Varian, H.R. (1993) Microeconomic Analysis, Third Edition. New York: Norton. 